\title{
Mid infrared polarisation of Ultraluminous Infrared Galaxies
}

\author{
R. Siebenmorgen ${ }^{1}$ and A. Efstathiou ${ }^{2}$
}

\author{
1 European Southern Observatory, Karl-Schwarzschildstr. 2, 85748 Garching b.München, Germany \\ 2 Astrophysics Group, Blackett Laboratory, Imperial College of Science Technology \& Medicine, \\ Prince Consort Rd., London. SW7 2BZ, UK
}

Received 25 May 2001 / Accepted 30 July 2001

\begin{abstract}
The mid infrared polarisation properties of four Ultraluminous Infrared Galaxies (ULIRGs) have been investigated by broad band filter observations with the ISOCAM instrument on board the Infrared Space Observatory (ISO). The wavelength region from 5 to $18 \mu \mathrm{m}$ was selected where the emission from the putative torus peaks. We report detection of polarisation for all ULIRGs studied. The fractional polarisation ranges from $\sim 3 \%$ up to $8 \%$. The highest polarisation is recorded in Mrk 231 which has a clear AGN signature, whereas the lowest is for Arp 220, which is generally thought to be powered predominantly by star formation. We discuss the various mechanisms that could give rise to the polarisation and conclude that the most likely interpretation is that it is due to magnetically aligned elongated dust grains. This is the same mechanism believed to be operating in a number of galactic sources. The position angle of polarisation could give the projected magnetic field direction and therefore constrain models for the formation of the tori.
\end{abstract}

Key words. polarisation - infrared: galaxies - galaxies: ISM - galaxies: individual: Mrk 231, Arp 220, IRAS $15250+3609$, Mrk 273

\section{Introduction}

One of the most surprising discoveries of the Infrared Astronomical Satellite (IRAS) was that of a local population of Ultraluminous Infrared galaxies (ULIRGs) with far IR luminosities $\left(L_{\mathrm{FIR}}>10^{12} L_{\odot}\right)$ and space densities higher than those of quasars (Sanders et al. 1988). The recent discovery of a large number of submillimeter galaxies, which could be primeval ULIRGs, with SCUBA (Hughes et al. 1998; Barger et al. 1998) and other far IR surveys (Puget et al. 1999; Kawara et al. 1998; Efstathiou et al. 2000), has renewed interest in this class of object.

The origin of the extreme luminosity of ULIRGs, is still a matter of controversy. While it is generally agreed that the IR luminosity is due to emission from dust that reprocesses optical and UV light to longer wavelengths, it is still not clear whether the primary source of radiation is a burst of star formation (e.g. Downes \& Solomon 1998; Skinner et al. 1997) or dust-enshrouded accreting black holes (Sanders et al. 1988; Soifer et al. 2000).

A number of observational techniques have been employed in an attempt to settle this issue. However, the high degree of obscuration makes traditional diagnostics such as optical and near IR (Goldader et al. 1995) emission

Send offprint requests to: R. Siebenmorgen,

e-mail: rsiebenm@eso.org

* Based on observations with ISO, an ESA project with instruments funded by ESA Member States (especially the PI countries: France, Germany, The Netherlands and the UK) with the participation of ISAS and NASA. lines difficult to interpret. Recently, mid IR spectroscopy which is less affected by obscuration, obtained with the ISO satellite, has shown that ULIRGs are predominantly powered by star formation (Genzel et al. 1998; Rigopoulou et al. 1999; Clavel et al. 2000). However, the optical depth of the dusty medium (believed to be in the form of a torus) that is obscuring the AGN may be optically thick to suppress even the mid IR lines.

Following the discovery of broad emission lines in the polarised flux of the prototypical narrow-line active galactic nucleus (AGN) NGC 1068 (Antonucci \& Miller 1985), a lot of effort has been directed towards the study of the polarisation properties of galaxies (Heisler et al. 1997). The generally accepted interpretation of this phenomenon is that our view of the sub-parsec scale region of ionized gas producing the broad emission lines is blocked by a geometrically and optically thick dusty torus. In this situation the broad lines are detected because they are reflected (and hence polarised) by free electrons along the axis of the torus. The postulated dusty torus is the central theme of the "unified model" for active galaxies which holds that a lot of the diversity in their observed properties is the result of the orientation of the observer with respect to the axis of the torus (Antonucci 1993).

An important corollary of the obscuring torus hypothesis is that a large fraction of the power emitted by an AGN is absorbed and re-emitted by dust in the IR. Models of the IR emission from such tori have been presented by a number of authors (Pier \& Krolik 1992; Granato \& Danese 1994; Efstathiou \& Rowan-Robinson 1995; 
Ruiz et al. 2001). The predicted emission peaks in the $3-30 \mu \mathrm{m}$ range and shows spectral features at $9.7 \mu \mathrm{m}$ due to silicate dust. However, since the torus emission is usually unresolved it is difficult to separate the emission of the torus from that of other dusty parts of the galaxy such as giant molecular clouds heated by young stars (Krügel \& Siebenmorgen 1994).

Until large IR telescopes/interferometers for directly imaging the torus become available its properties can only be probed by indirect methods, a very powerful of which promises to be IR polarimetry (Efstathiou et al. 1997). IR polarisation can of course be produced by non-thermal processes (synchrotron), and we discuss this later, but what is of more interest to us here is polarisation produced by the dichroic absorption (or emission) by non-spherical magnetically aligned grains. This effect is similar to the one that gives rise to the polarisation in the optical by interstellar grains aligned by the galactic magnetic field (Hall 1949; Hiltner 1949).

If dust grains are similarly aligned in the dusty torus by the magnetic field, which in some models is thought to be a prerequisite for its formation (Krolik \& Begelman 1988; Koningl \& Kartje 1994), then we expect the radiation emitted (and self-absorbed) by the torus to be polarised (Efstathiou et al. 1997). The region that produces the IR polarisation, the torus, is therefore different from the region that produces the optical and UV polarisation. The effect of polarisation due to magnetically aligned dust particles has been observed so far in a few galactic environments such as the Galactic Centre (Hildebrand 1989), molecular clouds (Novak et al. 1989; Clemens et al. 1999), star forming regions (Gonatas et al. 1990; Leach et al. 1991; Smith et al. 2000), protostars (Siebenmorgen \& Krügel 2000) and prestellar cores (Minchin et al. 1995; Ward-Thompson et al. 2000). Extended polarisation was recently reported for two external galaxies: M 82 (Greaves et al. 2000) and NGC 1808 (Siebenmorgen \& Krügel 2001). The latter authors argue against mid IR polarisation due to small grains such as PAHs or nanometre-sized silicates.

With the expectation of detecting the IR polarisation from dusty tori in ULIRGs, if they exist, we embarked on a program of mid IR polarimetry with the ISOCAM instrument (Cesarksy et al. 1996) on the Infrared Space Observatory (ISO, Kessler et al. 1996). During the ISO mission four of the ULIRGs in the IRAS Bright Galaxy sample $(60 \mu \mathrm{m}$ flux density $S(60)>5 \mathrm{Jy}$; Sanders et al. 1988) could be observed. Targets were selected mainly on visibility constraints so that they represent a random subset of the Sanders sample.

\section{Observations}

ISOCAM (Cesarsky et al. 1996) polarimetric imaging of the ULIRGs studied was set up according to the observing template CAM05 (Siebenmorgen et al. 1996). We used a $2 \times 2$ raster with a raster step size of $36^{\prime \prime}$ and a $6^{\prime \prime}$ pixel field of view. The read out time of each exposure was $2.1 \mathrm{~s}$. We took initially 60 exposures on the source to stabilize the detector and then performed a raster through the free hole of the entrance wheel. On each raster position of the hole measurements 15 exposures were read. Then three raster were taken by rotating the polarisers on each raster position. For each combination of polariser and raster position 23 exposures were read. The polariser rasters were repeated in 3 times. This procedure gives a total of twelve independent measurements for each polarisers.

We present broad band filter observations LW2, LW10 and LW3, centred at $6.7,12.0$ and $14.3 \mu \mathrm{m}$, at resolution of $5.4^{\prime \prime}, 9.6^{\prime \prime}$ and $11.4^{\prime \prime}$, respectively. The data are reduced with the ISOCAM interactive analysis system (CIA version 4.0, Ott et al. 1996). Only basic reduction steps, such as dark current subtraction, removal of cosmic ray hits, transient correction and flat fielding are applied (see Siebenmorgen et al. 2000 for more details on ISOCAM data). Photometry is done by integrating the flux in an aperture radius of 6 pixels. A background estimate is derived from the mean flux in a 4 pixel wide circular annulus 2 pixels away from the source aperture. The same procedure is applied on a model point spread function (Okumura et al. 1998) to correct the derived source fluxes for losses in higher airy rings. The dead column of the LW-detector was masked out. Instrumental polarisation is corrected by applying the polarisation weight factors as described by Siebenmorgen (1999).

For each measurement the polarised signal is found to be consistent between the cycles. The average of all cycles gives the final Stokes vector. The (unpolarised) standard star HIC 085371 was also observed with the same strategy as a test of our observing and data reduction procedure. We find a residual degree of polarisation of $p=0.45 \pm 0.4 \%$ for this star, which reflects the limit of our processing technique and calibration uncertainty. This observing mode and data reduction procedure has also been used to detect a $20 \%$ polarisation at $14.3 \mu \mathrm{m}$ in the Crab nebula at a position angle consistent with optical and radio measurements (Tuffs et al. 1999), thus providing a further test of the methods applied.

Results of the ISO observations together with near IR polarimetry by Jones \& Klebe (1989) of the four ULIRGs are summarised in Table 1. At the resolution of ISO the objects appear point like. The corresponding observing sequence or TDT numbers read for target: Mrk 231 (56800101, 56800201), Arp 220 (61900201, 61900301, 61900401), IRAS 15250+3609 (57400701, 57400801, 57400901) and Mrk 273 (52700101, 52700201, $52700301)$, respectively.

\section{Discussion}

For all ULIRGs we find significant polarisation in the range of $\sim 3-8 \%$. The highest polarisation is recorded in Mrk 231 which has a clear AGN signature from other studies (e.g. Genzel et al. 1998). The lowest polarisation is found for Arp 220, which is generally thought to be powered by star formation and where only very recently evidence for a hidden quasar was found (Haas et al. 2001). 
Table 1. Infrared polarisation of ULIRGs. Near IR data by Jones \& Klebe (1989). ISO data this work.

\begin{tabular}{|c|c|c|c|c|}
\hline Object & \begin{tabular}{|l|} 
Band \\
$\mu \mathrm{m}$
\end{tabular} & $\begin{array}{l}F \\
\text { mJy }\end{array}$ & $\begin{array}{l}p \\
\%\end{array}$ & $\begin{array}{l}\theta \\
\operatorname{deg}\end{array}$ \\
\hline \multirow[t]{5}{*}{ Mrk 231} & $J$ & & $0.77 \pm 0.14$ & $102 \pm 6$ \\
\hline & $H$ & & $0.62 \pm 0.12$ & $95 \pm 6$ \\
\hline & $K$ & & $0.46 \pm 0.08$ & $107 \pm 5$ \\
\hline & 12.0 & $989 \pm 7$ & $8.6 \pm 0.9$ & $123 \pm 4$ \\
\hline & 14.3 & $1719 \pm 10$ & $6.7 \pm 0.9$ & $126 \pm 4$ \\
\hline \multirow[t]{6}{*}{ Arp 220} & $J$ & & $1.15 \pm 0.25$ & $58 \pm 7$ \\
\hline & $H$ & & $0.58 \pm 0.16$ & $80 \pm 9$ \\
\hline & $K$ & & $0.54 \pm 0.18$ & $58 \pm 11$ \\
\hline & 6.7 & $272 \pm 3$ & $2.3 \pm 0.8$ & $50 \pm 10$ \\
\hline & 12.0 & $399 \pm 4$ & $2.6 \pm 0.9$ & $52 \pm 11$ \\
\hline & 14.3 & $794 \pm 7$ & $3.1 \pm 0.9$ & $62 \pm 9$ \\
\hline \multirow[t]{3}{*}{ IRAS $15250+3609$} & 6.7 & $84 \pm 2$ & $4.6 \pm 1.8$ & $153 \pm 10$ \\
\hline & 12.0 & $109 \pm 3$ & $6.3 \pm 1.5$ & $151 \pm 7$ \\
\hline & 14.3 & $200 \pm 5$ & $4.3 \pm 1.4$ & $164 \pm 9$ \\
\hline \multirow[t]{5}{*}{ Mrk 273} & $J$ & & $0.0 \pm 0.35$ & \\
\hline & $K$ & & $0.42 \pm 0.30$ & \\
\hline & 6.7 & $153 \pm 2$ & $6.2 \pm 3.0$ & $159 \pm 14$ \\
\hline & 12.0 & $210 \pm 3$ & $6.7 \pm 1.3$ & $174 \pm 6$ \\
\hline & 14.3 & $361 \pm 5$ & $6.5 \pm 1.0$ & $171 \pm 4$ \\
\hline
\end{tabular}

In Mrk 231 we can rule out a synchrotron interpretation as the extrapolated non-thermal emission at $1 \mathrm{~mm}$ (Downes \& Solomon 1998) is contributing less than $1 \%$ of the $12 \mu \mathrm{m}$ emission. Also dust scattering should not be an important mid IR polarisation mechanism since the grains are tiny compared to the wavelength and their scattering cross sections are very small (Smith et al. 1995). Therefore the mid IR polarisation of Mrk 231 is most likely due to dichroism of magnetically aligned spheroidal dust grains. Carilli et al. (1998) presented high resolution radio images of Mrk 231 which indicate the existence of a subkiloparsec disc with its major axis oriented east-west and viewed at an orientation of about $45^{\circ}$. On an even smaller scale mapped with VLBI, there is evidence for a compact core and a radio lobe, about $20 \mathrm{pc}$ from the core, with the axis of the core-lobe system being approximately perpendicular to the major axis of the sub-kiloparsec disc. The position angle of the polarisation is approximately parallel to the major axis of the sub-kiloparsec disc. If we assume that the rotation axis of the putative dusty torus in Mrk 231 and the sub-kiloparsec disc are the same and the magnetic field lies in the plane of the disc (as in other disc like systems like the Milky Way), then we expect the position angle of polarisation produced by dichroic absorption to be parallel to this magnetic field direction as observed. We cannot decide on the basis of these observations alone whether the polarisation is emissive or absorptive although the fact that the polarisation angle is parallel to the plane of the disc is tempting us to favor the latter mechanism. With spectro-polarimetry across the silicate feature one should be able to discriminate between the two dichroic polarisation mechanisms. Jones \& Klebe (1989) argue that the wavelength dependence of the optical to near IR polarisation of Mrk 231 is not consistent with a screen of aligned grains nor with synchrotron radiation. They favor scattering with dilution as the prime polarisation mechanism. The source of dilution in the optical could be starlight whereas in the near IR may be due to a power-law component. We note that scattering of UV to near IR radiation by dust flowing outward along the North-South VLBI axis would give rise to polarisation with a position angle of roughly $90^{\circ}$. This could explain the relative agreement between the optical and mid IR position angles despite the fact that the polarisation mechanism is different.

For Arp 220, Jones \& Klebe (1989) argue that the near IR polarisation is due to extinction through aligned grains. The main argument is that the wavelength dependence of polarisation is consistent with normal interstellar polarisation as known from the Milky Way. The position angle of polarisation in Arp 220 is aligned with the position angle of the $5^{\prime \prime} \times 1.6^{\prime \prime} \mathrm{CO}$ disc observed by Downes \& Solomon (1998). The polarisation angle follows also the dust lane seen in the optical. If the magnetic field structure is parallel to the disc, such an orientation of the polarisation angle is expected if we interpret the detected 6-15 $\mu \mathrm{m}$ polarisation as due to dichroic absorption by aligned dust grains in the disc. ISO observations and models (Siebenmorgen et al. 1999) of Arp 220 result in high dust extinction estimates of $A_{V} \sim 54 \mathrm{mag}$ to the center of the disc. This may be converted to a $14 \mu \mathrm{m}$ optical depth of $\tau_{14 \mu \mathrm{m}} \sim 2.5$ using extinction profiles (e.g. Fig. 12 in Krügel \& Siebenmorgen 1994). With perfectly aligned spinning spheroids of silicate or amorphous carbon, and for such high optical depths, the degree of mid IR polarisation is expected to be high (Siebenmorgen \& Krügel 2000). On the contrary, the observed polarisation is low, a fact which points to inefficient grain alignment expected for extremely disordered magnetic field structures. A similar interpretation is given by Jones \& Klebe (1989) for the low near IR polarisation.

IRAS $15250+3609$ is classified as a LINER by Veilleux et al. (1995). Sopp \& Alexander find a North-South extension in their $15 \mathrm{GHz}$ map, i.e. with roughly the same position angle as our polarisation measurement. This again may give some indication to favor dichroic absorption as the dominant mid IR polarisation mechanism.

Mrk 273 has a double nucleus with at least one of its nuclei known to contain a type II AGN (e.g. Sargent 1972). In the mid IR the source has been resolved into two components which contribute about equal amounts to the total flux (Soifer et al. 2000). The northern component shows a deeper silicate absorption feature than the southern source and is also associated with a CO source which Downes \& Solomon (1998) resolved into a compact source and a disc oriented East-West. The position angle of this disc is perpendicular to the position angle of the mid IR polarisation. This may give some indication that the mid IR polarisation is due to dichroic emission. Also we note that at a distance of $\sim 150 \mathrm{Mpc}$ we probe only the 
innermost $2 \mathrm{kpc}$ region of the nuclei and mid IR polarimetry at higher resolution is certainly required to clarify the situation.

The IR polarisation due to aligned dust grains shows, except for the silicate band, a relative flat dependence with wavelength. So that galaxies which show dichroic mid IR polarisation should also be polarised in the far IR and submillimeter. Then according to the dichroic model the far-IR emission should be polarised with a position angle perpendicular to that seen in absorption. This effect has been observed by Hildebrand et al. (1984) in Orion. However, the far IR/submm emission may well have significant contribution from star burst activity or from the host galaxy so that the mid IR is probably the best range to probe the AGN.

Acknowledgements. CIA is a joint development by the ESA Astrophysics Division and the ISOCAM Consortium. The ISOCAM Consortium is led by the ISOCAM PI, C. Cesarsky.

\section{References}

Antonucci, R. R. J. 1993, ARA\&A, 31, 473

Antonucci, R. R. J., \& Miller, J. S. 1985, ApJ, 297, 621

Barger, J. A. J., Cowie, L. L., Sanders, D. B., et al. 1998, Nature, 394, 248

Carilli, C. L., Wrobel, J. M., \& Ulvestad, J. S. 1998, AJ, 115, 928

Cesarsky, C., Abergel, A., Agnèse, P., et al. 1996, A\&A, 315, L32

Clavel, J., Schulz, B., Altieri, B., et al. 2000, A\&A, 357, 839

Clemens, D., Kraemer, K., \& Ciardi, D. 1999, ISO Polarisation Observations, ed. R. J. Laureijs, \& R. Siebenmorgen, ESASP435, ISBN 92-9092-740-2,

http://www.iso.vilspa.esa.es

Downes, D., \& Solomon, P. M. 1998, ApJ, 507, 615

Efstathiou, A., \& Rowan-Robinson, M. 1995, MNRAS, 273, 649

Efstathiou, A., McCall, A., \& Hough, J. H. 1997, MNRAS, 285, 102

Efstathiou, A., Oliver, S., Rowan-Robinson, M., et al. 2000, MNRAS, 319, 1169

Gonatas, D. P., Engargiola, G. A., Hildebrand, R. H., et al. 1990, ApJ, 357, 132

Goldader, J. D., Joseph, R. D., Doyon, R., \& Sanders, D. B. 1995, ApJ, 444, 97

Granato, G. L., \& Danese, L. 1994, MNRAS, 268, 235

Greaves, J., Holland, W. S., Jenness, T., et al. 2000, Nature, 404,732

Genzel, R., Lutz, D., Sturm, E., et al. 1998, ApJ, 498, 579

Haas, M., Klaas, U., Müller, S. A. H., Chini, R., \& Coulson, I. 2001, A\&A, 367, 9

Hall, J. S. 1949, Science, 109, 166

Heisler, C. A., Lumsden, S. L., \& Bailey, J. A. 1997, Nature, 385,700

Hildebrand, R. H., Dragovan, M., \& Novak, G. 1984, ApJ, 284, L51

Hildebrand, R. H. 1989, Interstellar Dust, ed. L. J. Allamandola, \& A. G. G. M. Tielens (IAU), 275

Hiltner, W. A. 1949, Science, 109, 165

Hughes, D. H., Serjeant, S., Dunlop, J., et al. 1998, Nature, 394,241
Jones, T. J., \& Klebe, D. 1989, ApJ, 341, 707

Kawara, K., Sato, Y., Matsuhara, H., et al. 1998, A\&A, 336, L9

Kessler, M. F., Steinz, J. A., Anderegg, M. E., et al. 1996, A\&A, 315, L27

Koningl, A., \& Kartje, J. 1994, ApJ, 434, 446

Krolik, J., \& Begelman, M. C. 1988, ApJ, 329, 702

Krügel, E., \& Siebenmorgen, R. 1994, A\&A, 288, 929

Leach, R. W., Clemens, D. P., Kane, B. D., et al. 1991, ApJ, 370,257

Minchin, N. R., Sandell, G., \& Murray, A. G. 1995, A\&A, 293, L61

Novak, G., Gonatas, D. P., Hildebrand, R. H., et al. 1989, ApJ, 345,802 .

Okumura, K. 1998, ISOCAM PSF Report, available at: http://www.iso.vilspa.esa.es/users/expl_lib/ CAM_list.html

Ott, S., Abergel, A., Altieri, B., et al. 1996, ASP Conf. Ser., 125, 1997

Pier, E. A., \& Krolik, J. H. 1992, ApJ, 401, 99

Puget, J.-L., Lagache, G., Clements, D. L., et al. 1999, A\&A, 345,29

Rigopoulou, D., Spoon, H. W. W., \& Genzel, R. 1999, AJ, 118, 2625

Ruiz, M., Efstathiou, A., Alexander, D. M., et al. 2001, MNRAS, in press

Sanders, D. B., Soifer, B. T., Elias, J. H., et al. 1988, ApJ, 325, 74

Sargent, W. L. W. 1972, ApJ, 173, 7

Siebenmorgen, R. 1996, Polarimetric imaging with ISOCAM: C05 Observer's Manual, ESA/SAI/96-238/Dc, http://www.iso.vilspa.esa.es

Siebenmorgen, R. 1999, ISO Polarisation Observations, in Observing Polarisation with the Infrared Space Observatory, ed. R. J. Laureijs, \& R. Siebenmorgen, ESASP435, ISBN 92-9092-740-2, http://www.iso.vilspa.esa.es

Siebenmorgen, R., Krügel, E., \& Zota, V. 1999, A\&A, 351, 140

Siebenmorgen, R., Blommaert, J., Sauvage, M., \& Starck, J. L. 2000, ISO Handbook, vol. III (CAM), Version 1.0, ESA document, Reference: SAI/99-057/Dc,

http://www.iso.vilspa.esa.es/users/expl_lib/

CAM_top.html

Siebenmorgen, R., \& Krügel, E. 2000, A\&A, 364, 625

Siebenmorgen, R., \& Krügel, E. 2001, submitted to A\&A

Skinner, C. J., Smith, H. A., Sturm, E., et al. 1997, Nature, 386,472

Smith, C. H., Wright, C. M., Aitken, D. K., et al. 2000, ApJ, 312,327

Smith, P. S., et al. 1995, ApJ, 444, 146

Soifer, B. T., Neugebauer, G., Matthews, K., et al. 2000, AJ, 119,509

Sopp, H. M., \& Alexander, P. 1991, MNRAS, 251, 112

Tuffs, R. J., Siebenmorgen, R., \& Gallant, Y. A. 1999, MIR polarimetric mapping of the Crab Nebula, in Observing Polarisation with the Infrared Space Observatory, ed. R. J. Lauriejs, \& R. Siebenmorgen, ESA-SPC, available at: http://www.iso.vilspa.esa.es/meetings/polarisation/ paper/web/

Ward-Thompson, D., Kirk, J. M., Crutcher, R. M., et al. 2000, ApJ, 537, L135

Veilleux, S., Kim, D. C., Sanders, D. B., Mazzarella, J. M., \& Soifer, B. T. 1995, ApJS, 98, 171 